\title{
LA VARIABILIDAD DE LA FRECUENCIA CARDÍACA
}

\footnotetext{
Oomo se pudo ver en los capítulos anteriores, la frecuencia cardíaca es un parámetro que se puede medir a partir de la detección de una característica particular de la señal ECG. El parámetro detectado corresponde al tiempo entre cada onda R de la señal ECG, denominado tiempo RR. A partir de este valor de tiempo se construye un arreglo de datos que toma el nombre de señal de ritmo cardíaco.

La construcción de esta señal se puede explicar fácilmente tomando como ejemplo una función matemática discreta $\mathrm{x}\left(\mathrm{t}_{\mathrm{n}}\right)$. Donde $\mathrm{x}\left(\mathrm{t}_{\mathrm{n}}\right)$ contiene el valor de tiempo entre cada onda $R$ de la señal ECG. El dato $t_{n}$ corresponde al tiempo acumulado y se puede estimar a partir de los tiempos RR.

El propósito de este capítulo es ofrecer una introducción a los métodos matemáticos básicos para la extracción de información de la señal de ritmo cardíaco. En este sentido, serán expuestos métodos clasificados en dos grandes grupos: análisis en el dominio del tiempo y análisis en el dominio de la frecuencia.
}

La figura 50 contiene un ejemplo gráfico de una señal de ritmo cardíaco con sus respectivas unidades. Se puede observar que para cada valor de 
tiempo $R R$ se posee un valor $t$ y esto ofrece una característica irregular en el tiempo. Cada valor de tiempo RR almacenado en la variable $x\left(t_{n}\right)$ tiene su aparición en el instante $t$.

FIGURA 50. LA SEÑAL DE RITMO CARDÍACO

FUENTE:

ELABORACIÓN PROPIA.

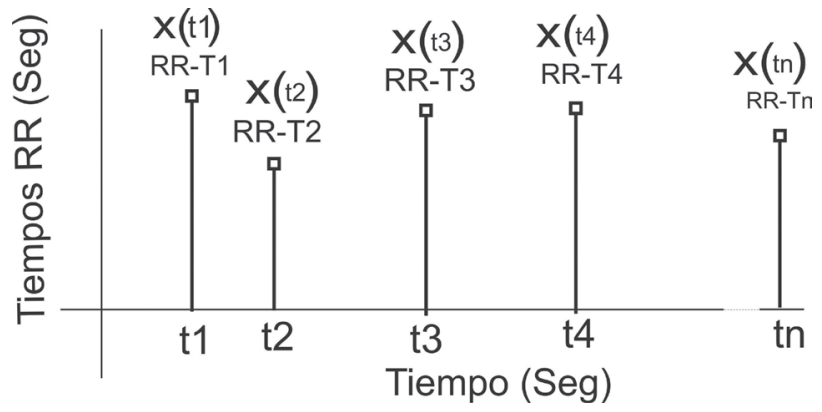

La variable $t_{n}$ puede modelarse a través de la ecuación 8 .

ECUACIÓN 8.

$$
t_{n}=x_{1}\left(t_{1}\right)+x_{2}\left(t_{2}\right)+\cdots \ldots+x_{n-2}\left(t_{n-1}\right)
$$

A través del asistente matemático Matlab es posible tomar un grupo de valores de tiempo $R R$, almacenados en la variable $R R 1$, y construir la señal de ritmo cardíaco. El propósito del siguiente código propuesto es generar la base de tiempo, la cual está acorde con la ecuación 8 y determina la ocurrencia de cada muestra. El código permite generar la variable $t$, que contiene la base de tiempo irregular para cada muestra del arreglo RR1.

$\%$ Arreglo que contiene la base de tiempo: RR1

$N=$ length (RR1);

$\%$ Inicio de variable acumuladora

$$
\begin{aligned}
& c=0 ; \\
& \text { fori }=1: N \\
& t(i)=c+R R 1(i) ; \\
& c=t(i) ;
\end{aligned}
$$


$\operatorname{plot}(t, R R 1)$

title('Señal de Ritmo cardíaco)

xlabel('Tiempo (Seg)')

ylabel(' Tiempo $R-R(\operatorname{Seg})$ ')

La señal de ritmo cardíaco es el soporte de todas las investigaciones basadas en el análisis de la variabilidad de la frecuencia cardíaca. De acuerdo con la literatura, la señal de ritmo cardíaco es el insumo de dos tipos de procedimientos de los cálculos de índices estadísticos en el dominio del tiempo y de la frecuencia.

\section{Análisis en el dominio del tiempo}

El análisis en el dominio del tiempo consiste en el cálculo de índices estadísticos a partir de la señal de ritmo cardíaco. El propósito de este análisis es encontrar tendencias del comportamiento de los cambios de frecuencia cardíaca instantánea. La tabla 1 contiene un listado de variables que representan los principales índices estadísticos calculados para el análisis en el dominio del tiempo.

TABla 1. PARÁmetros MEDIDOS EN EL ANÁLISIS DEL DOMINIO DEL TIEMPO

\begin{tabular}{|c|c|c|c|}
\hline Varible & Unidad & Descripción & Fórmula \\
\hline SDNN & $\mathrm{ms}$ & $\begin{array}{l}\text { Desviación estandar de los } \\
\text { intervalos RR. }\end{array}$ & $\sigma_{R R}=\sqrt{\frac{1}{N-1} \sum_{i=1}^{N}(R R(i)-R R)^{2}}$ \\
\hline RMSSD & $\mathrm{ms}$ & $\begin{array}{l}\text { Raíz cuadrada del promedio } \\
\text { de las suma de las diferencias } \\
\text { entre intervalos RR } \\
\text { adyacentes. }\end{array}$ & 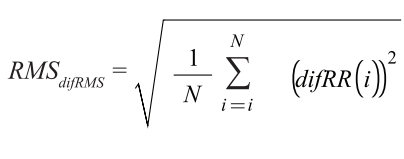 \\
\hline NN50 & $\mathrm{n}$ & $\begin{array}{l}\text { Número de pares de intervalos } \\
\text { RR adyacentes cuya diferencia } \\
\text { es de más de } 50 \mathrm{~ms} \text {. }\end{array}$ & $(R R(i)-R R(i+1))>50 \mathrm{~ms}$ \\
\hline pNN50 & $\%$ & $\begin{array}{l}\text { Porcentaje del conteo de NN50 } \\
\text { de todos los intervalos RR: } \\
\text { (conteo de Nn50/conteo total } \\
\text { de los intervalos RR)x100. }\end{array}$ & $\%(R R(i)-R R(i+1))>50 \mathrm{~ms}$ \\
\hline
\end{tabular}

FUENTE: ELABORACIÓN PROPIA. 
Por lo general, los cálculos presentados son realizados tomando los tiempos RR de trazos electrocardiográficos en grabaciones de alrededor de 5 minutos. Cuando los cálculos son realizados en este tipo de grabaciones, toman el nombre de análisis de la variabilidad de la frecuencia cardíaca en corto tiempo. En el caso de análisis realizados en grabaciones de 24 horas, se denomina de largo tiempo.

\section{Análisis en el dominio de la frecuencia}

De manera complementaria al análisis en el dominio del tiempo, es posible estudiar la variabilidad de la frecuencia cardíaca a través de la densidad espectral de potencia (PSD). La medida de la PSD puede tener un grado de confiabilidad relativo, ya que la señal ECG y los tiempos RR no son funciones estacionarias.

La PSD ofrece como resultado una curva como la que se puede apreciar en la figura 51. La curva del PSD posee un ancho de banda de entre 0 y $1 \mathrm{~Hz}$, y está dividida en diferentes bandas de frecuencia [27].
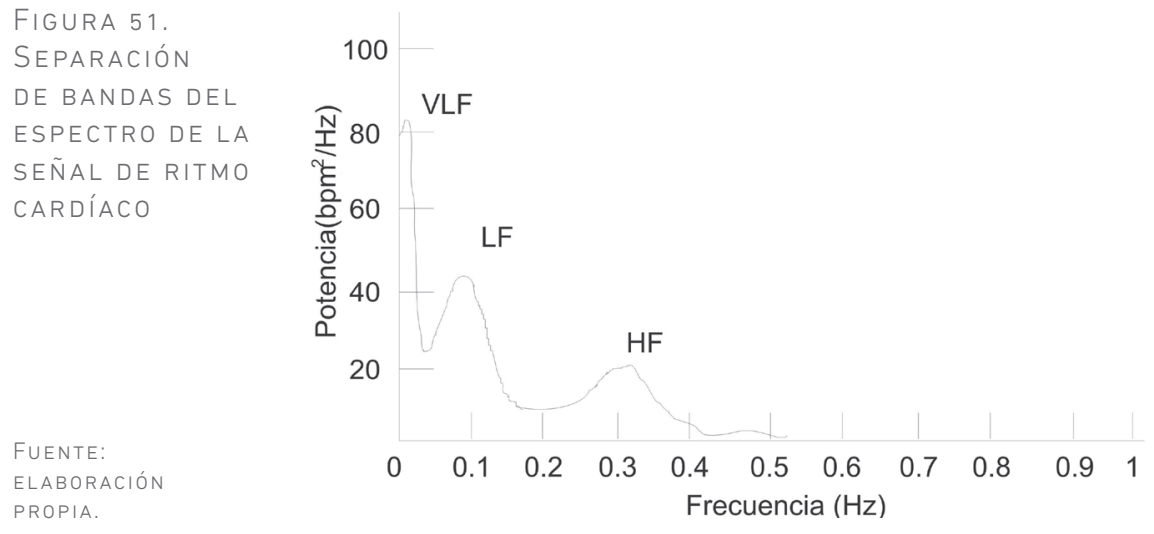

Entre las frecuencias de 0.15 y $0.4 \mathrm{~Hz}$, se puede encontrar la banda de altas frecuencias (HF). Esta banda está relacionada con los cambios de frecuencia cardíaca que son influenciados por el ciclo respiratorio. Se considera que la HF está estrechamente relacionada con el control que ejerce el sistema parasimpático. 
Seguidamente está presente la banda LF (bajas frecuencia) que comprende las frecuencias entre 0.04 y $0.15 \mathrm{~Hz}$. Esta banda está estrechamente relacionada con el comportamiento del sistema simpático, que es el encargado de aumentar la frecuencia de latidos del corazón.

La banda VLF (muy baja frecuencia) contiene las componentes entre 0 y $0.04 \mathrm{~Hz}$. Actualmente sigue bajo estudio el significado fisiológico de esta banda de frecuencia. Se mantiene la teoría de que la VLF está asociada a la termorregulación.

Con base en los resultados obtenidos al estimar la PSD, es posible calcular índices para el análisis en el dominio de la frecuencia. La tabla 2 contiene, para cada banda del espectro, los índices que son calculados para el análisis en el dominio de la frecuencia.

TABLA 2. PARÁMETROS MEDIDOS EN EL ANÁLISIS DEL DOMINIO DE LA FRECUENCIA

\begin{tabular}{lcll}
\hline Potencia VLF & ms2 & $<0.04 \mathrm{~Hz}$ & $\begin{array}{l}\text { Potencia de las componentes de muy baja } \\
\text { frecuencia. }\end{array}$ \\
\hline Potencia LF & ms2 & $0.04-0.15 \mathrm{~Hz}$ & Potencia de las componentes de baja frecuencia. \\
\hline LFnu & n.u. & & $\begin{array}{l}\text { Potencia LF en unidades normalizadas. Potencia } \\
\text { LF/(potencia total - potencia VLF) x100. }\end{array}$ \\
\hline Potencia HF & ms2 & $0.15-0.4 \mathrm{~Hz}$ & Potencia de las componentes de alta frecuencia. \\
\hline Hfnu & n.u. & & $\begin{array}{l}\text { Potencia HF en unidades normalizadas. Potencia } \\
\text { HF/(potencia total - potencia VLF) x100. }\end{array}$ \\
\hline LF/HF & & Relación de LF a HF. Potencia LF/potencia HF. \\
\hline
\end{tabular}

FUENTE: ELABORACIÓN PROPIA.

Para la estimación de la PSD de la señal de ritmo cardíaco, es necesario tener en cuenta que esta serie de tiempo posee un muestreo irregular. Esto hace que el uso de la transformada de Fourier no puede ser de forma directa.

Tradicionalmente, se aplica un proceso de interpolación y remuestreo antes de la estimación de la PSD. La figura 52 ilustra la metodología para la estimación de la PSD teniendo en cuenta la interpolación. 
FIGURA 52. MÉTODO TRADICIONAL PARA LA ESTIMACIÓN DE LA PSD DE LA SEÑAL DE RITMO CARDÍACO

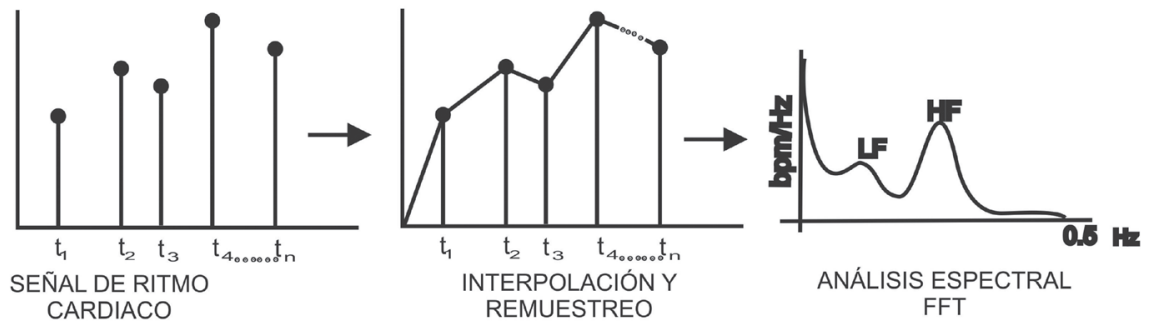

FUENTE:

ELABORACIÓN

PROPIA.

Por medio del asistente matemático Matlab se puede diseñar una estrategia para ejecutar la metodología de la figura 52. En las siguientes líneas de código se toma el arreglo de datos RR1, que contiene la señal de ritmo cardíaco. Se establece el valor más pequeño de tiempo que posee para estimar un periodo de muestreo óptimo.

$$
\begin{aligned}
& \text { Ts=min(RR1)/4; } \\
& \text { Fs=1/Ts; } \\
& \text { tfinal=t(N); } \\
& \text { ti=t(1):Ts:tfinal; } \\
& x=\text { interp1( } t, R R 1, \text { ti,'linear'); } \\
& N 1=\text { length }(x) ;
\end{aligned}
$$

figure

$$
\operatorname{plot}(t i, x)
$$$$
\text { xlabel('Tiempo(Seg)') }
$$$$
\text { ylabel(Tiempos } R-R \text { (Seg)') }
$$

El código proporciona como salida el arreglo de datos x que contiene una versión interpolada y con nuevo muestreo de la señal de ritmo cardíaco. La figura 53 contiene el resultado obtenido. 
FIGURA 53.

SEÑAL DE

RITMO CARDÍACO

INTERPOLADA

FUENTE:

ELABORACIÓN

PROPIA.

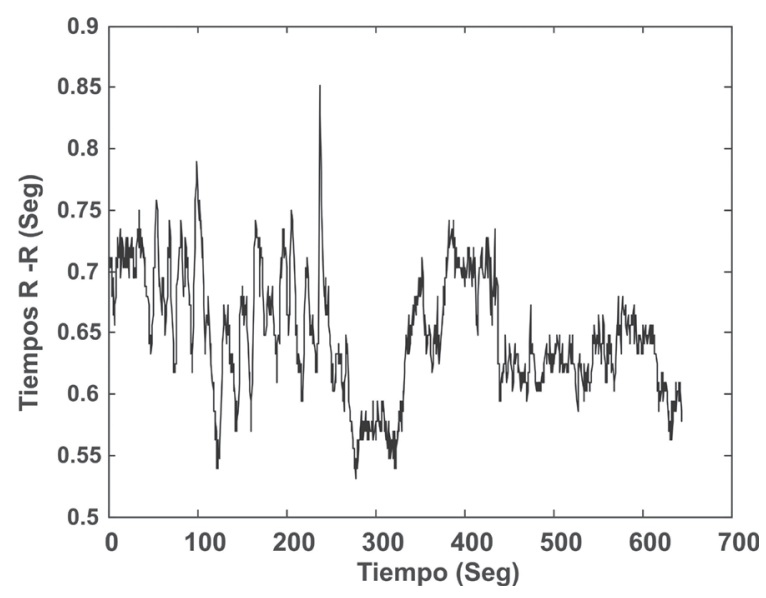

La siguiente parte del código contiene la ejecución del enventando y estimación de la PSD a través del algoritmo de la transformada rápida de Fourier:

$W=\operatorname{hamming}(\mathrm{N1})$;

$x f=\operatorname{abs}\left(f f t\left(x \cdot{ }^{*} W^{\prime}\right)\right)$;

$x f=x f(1: N 1 / 2)$;

$k=1: N 1 / 2$;

$F=(k-1) * F s / N 1$;

$x f=x f / \max (x f)$;

figure

$\operatorname{plot}(F, \log 10(x f))$

La figura 54 presenta el resultado obtenido al estimar la PSD de la señal de ritmo cardíaco. Se puede apreciar un efecto pasa bajo a partir de las componentes HF.

Para contrarrestar el efecto pasa bajo se ha propuesto un método alternativo basado en la estrategia de Lomb. Este método parte de la adquisición de un arreglo de datos $\mathrm{x}(\mathrm{t})$, el cual contiene las muestras adquiridas para cada tiempo $t$ y calcula la densidad espectral de potencia para cada valor de frecuencia $f$. 
FIGURA 54.

ESTIMACIÓN

DE LA PSD A

TRAVÉS DE LA

FFT EJECUTADA

SOBRE LA

SEÑAL DE

RITMO CARDÍACO

INTERPOLADA

Y CON NUEVO

MUESTREO

FUENTE:

ELABORACIÓN

PROPIA.

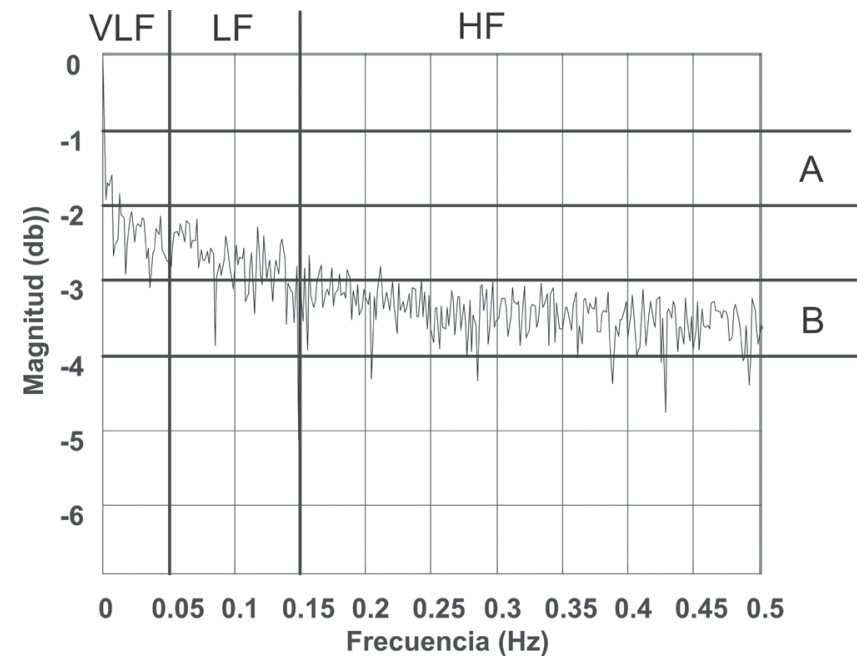

ECUACIÓN 9

$$
p(f)=\frac{1}{2 \delta^{2}}\left[\frac{\left(\sum_{n=1}^{N}\left(x_{n}\left(t_{n}\right)-x^{-}\right) \cdot \cos \left(2 \pi f\left(\left(t_{n}\right)-\tau\right)\right)\right)^{2}}{\sum_{n=1}^{N} \cos ^{2}\left(2 \pi f\left(\left(t_{n}\right)-\tau\right)\right)}+\frac{\left(\sum_{n=1}^{N}\left(x_{n}\left(t_{n}\right)-x^{-}\right) \cdot \sin \left(2 \pi f\left(\left(t_{n}\right)-\tau\right)\right)\right)^{2}}{\sum_{n=1}^{N} \sin ^{2}\left(2 \pi f\left(\left(t_{n}\right)-\tau\right)\right)}\right]
$$

En la ecuación $9, \delta$ corresponde a la varianza de los datos $\mathrm{x}(\mathrm{t})$ y el cálculo de la variable $\tau$ se realiza a través de la ecuación 10. La variable $\tau$ corresponde a una corrección aplicada a cada valor de tiempo $t$ debido a la condición irregular del tiempo de ocurrencia.

ECUACIÓN 10.

$$
\tan (4 \pi \tau)=\frac{\sum_{n=1}^{N} \sin \left(4 \pi \tau_{n}\right)}{\sum_{n=1}^{N} \cos \left(4 \pi \tau_{n}\right)}
$$


La figura 55 ilustra el resultado obtenido al aplicar la técnica de Lomb. En este caso se puede obtener una gran ventaja, que consiste en la ausencia del bloque de interpolación y remuestreo.

FIGURA 55. ESTIMACIÓN DE LA PSD A TRAVÉS DEL MÉTODO DE LOMB

FUENTE:

ELABORACIÓN PROPIA.

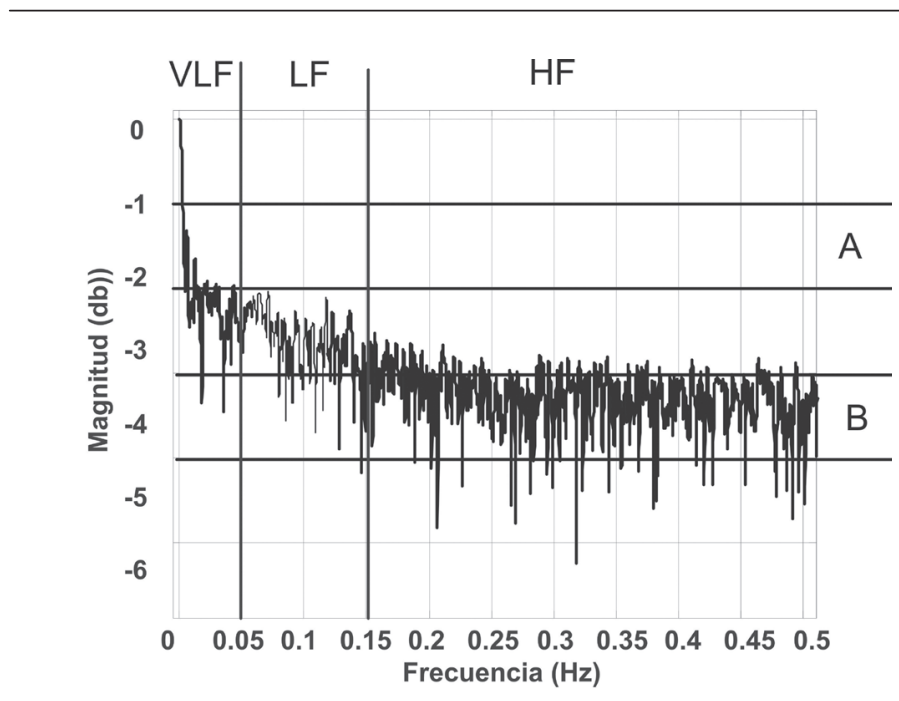

Por último, y a modo de recomendación, se ilustra en la figura 56 una comparación entre diferentes métodos para el análisis en el dominio de la frecuencia de la variabilidad de la frecuencia cardíaca. Esta comparación permite establecer que es posible escoger entre dos métodos diferentes.

Los métodos alternativos para la estimación de la PSD se encuentran actualmente en estudio. Estos métodos (figura 56) ofrecen la oportunidad de disminuir el esfuerzo computacional en cuanto a que no requieren una nueva interpolación de muestras para obtener una serie de tiempo con periodo de muestreo regular.

En resumen, este capítulo ha permitido al lector identificar que la variabilidad de la frecuencia cardíaca corresponde al cálculo de un conjunto de índices estadísticos. Estos cálculos pueden ser encontrados en el dominio del tiempo y de la frecuencia de la señal de ritmo cardíaco.

La intención de este capítulo no es solo mostrar los métodos clásicos y alternativos, también es una forma de dejar una puerta abierta para el planteamiento de investigaciones que permitan reducir la cantidad de cálculos matemáticos. 
FIGURA 56.

MÉTODOS

ALTERNATIVOS

PARA LA

ESTIMACIÓN DE

LA PSD DE LA

SEÑAL DE RITMO

CARDÍACO

\section{FUENTE:}

ELABORACIÓN

PROPIA.
ANÁLISIS DOMINIO

DE LA FRECUENCIA

SEÑAL DE

RITMO CARDIACO
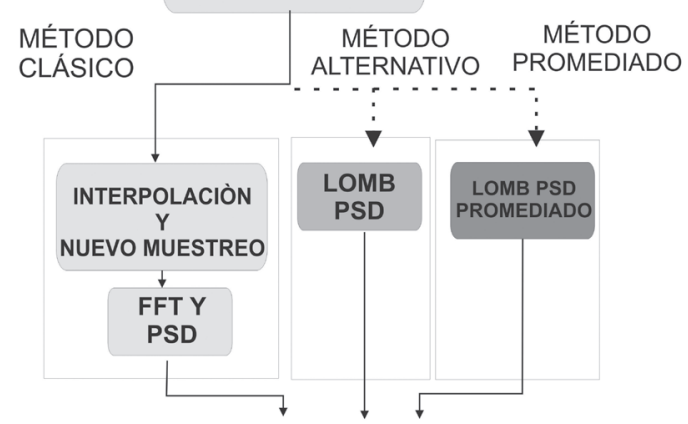

CÁLCULO DE

PARÁMETROS 


\section{CONCLUSIONES}

$\mathrm{E}^{1}$ procesamiento digital de señales es un área de la ingeniería que ha $\mathcal{C}_{\text {permitido generar estrategias para extraer información de las señales }}$ electrofisiológicas. Con respecto a la señal ECG, estos aportes han sido utilizados para dos fines específicos: atenuar distorsiones y permitir la detección de ondas características de la señal.

Se ha comprobado que los filtros digitales FIR son una herramienta útil para remover las distorsiones causadas por el desplazamiento de línea de base y línea de potencia eléctrica del electrocardiograma. Esta herramienta funciona solo cuando las causas de las distorsiones mantienen constantes sus parámetros.

Como alternativa de estrategias para controlar las distorsiones causadas por situaciones dinámicas, se han propuesto los sistemas de filtrado adaptativo. Estas estrategias permiten una flexibilidad en la función de transferencias de los filtros, la cual cambia a medida que la causa de la distorsión también lo hace.

El paso posterior al proceso de obtener una señal ECG libre de ruidos, consiste en implementar estrategias para la detección de la onda R. Los 
bancos de filtros permiten, de manera iterativa, encontrar un rango de frecuencias con las componentes espectrales asociadas a la onda R. Al poder realizar la detección de la onda $\mathrm{R}$, se obtiene la posibilidad de construir la señal de Ritmo cardíaco. 


\section{GLOSARIO}

Ag: símbolo de la tabla periódica para el elemento plata.

$\mathrm{AgCl}$ : Cloruro de Plata, componente esencial de los electrodos.

ADC: conversor análogo a digital, dispositivo que permite tomar señales analógicas en digitales.

Acondicionamiento: sistema analógico que permite adquirir, amplificar y filtrar una señal.

Aliasing: señales distorsionadas por causa de una mala selección de la frecuencia de muestreo.

ARM: tipo de dispositivo digital programable que se caracteriza por tener un conjunto reducido de instrucciones.

Bluetooth: protocolo para comunicaciones inalámbricas.

Capacitivo: que tiene la capacidad de acumular carga con un nivel de diferencia de potencial constante.

Conectividad: capacidad que posee un dispositivo de compartir datos en diferentes formatos y protocolos.

Derivaciones: diferentes conexiones realizadas sobre un sujeto para adquirir señales electrofisiológicas. 
Distorsión: cambios ocurridos en la morfología de una señal.

Descriptor: conjunto de variables que permiten detectar un parámetro o estado de una señal.

Fetal: alusivo a un ser vivo en proceso de gestación y que se encuentra en el vientre materno.

Filtro analógico: circuito electrónico para transformar señales analógicas o continuas en el tiempo.

Filtro digital: algoritmo basado en ecuaciones en diferencia que transforma una señal almacenada en un arreglo de datos.

Filtro digital adaptativo: versión robusta de un filtro digital que posee la capacidad de modificar su función de transferencia

Frecuencia: medida en ciclos por segundo y determina la velocidad de una señal

Frecuencia de muestreo: unidad que determina la cantidad de muestras por segundo por medio de la cual se discretiza una señal.

ECG: electrocardiograma. Se refiere a la señal eléctrica que caracteriza el comportamiento del corazón en seres humanos y animales mamíferos.

Espectro: curva obtenida en función del eje de las frecuencias y permite analizar la composición interna de una señal.

Electrodo: elemento que permite captar cambios de tensión en tejidos y convertirlos en señal eléctrica.

Interpolación: método matemático para estimar valores contenidos entre datos adquiridos de una señal.

Micro controlador: circuito electrónico que tiene la capacidad de cargar y ejecutar un programa en lenguaje propio. Es el conjunto de un procesador, memoria digital, bus de datos y puerto de comunicaciones.

Procesador: sistema digital que constituye el centro de un microcontrolador. Tiene la capacidad de ejecutar operaciones matemáticas a alta velocidad. 
Plataforma: conjunto de dispositivos electrónicos interconectados entre sí, orientados al desarrollo de equipos de procesamiento y medición de señales.

Ruido: señal que contiene datos que distorsiona la información de otras señales.

Señal: conjunto de valores tabulados con respecto a una variable independiente.

Transformada: procedimiento que permite la comparación de una señal con respecto a una función matemática preestablecida. 



\section{REFERENCIAS}

[1] W, Einthoven, “The telecardiogram”, American Heart Journal, vol. 53, núm. 4, pp. 602-615, 1957.

[2] C. Cooper; E. Clark, \& P.W., Macfarlane, "Enhanced detection of electrode placement/connection errors", Computers in Cardiology, vol.1, núm.1, pp. 89-92, 14-17, Septiembre 2008.

[3] T. Maruyama; M. Makikawa; N. Shiozawa \& Y. Fujiwara, “ECG Measurement Using Capacitive Coupling Electrodes for Man-Machine Emotional Communication", Complex Medical Engineering, CME 2007. IEEE/ ICME International Conference, vol. 1, núm. 1, pp. 378-383, 23-27 Mayo 2007.

[4] B.A. Reyes; H.F. Posada-Quintero; J.R. Bales \& K.H. Chon, “Performance evaluation of carbon black based electrodes for underwater ECG monitoring", Engineering in Medicine and Biology Society (EMBC), 2014 36th Annual International Conference of the IEEE, vol. 1, núm. 1, pp.1691-1694, Agosto 2014

[5] H.F. Posada-Quintero; B.A. Reyes; J.R. Bales; A.L. Clement; G.D. Pins; A. Swiston; J. Riistama; J.P. Florian; B. Shykoff; M. Qin \& K.H. Chon, 
"Novel Electrodes for Underwater ECG Monitoring”, Biomedical Engineering, IEEE Transactions on , vol. 61, núm. 6, pp. 1863-1876, Junio 2014.

[6] I. Pomasic \& R. Trobec, "Electrocardiographic Systems With Reduced Numbers of Leads-Synthesis of the 12-Lead ECG", Biomedical Engineering, IEEE Transactions, vol. 7, núm, pp.126-142, 2014.

[7] A. De Micheli-Serra \& P. Iturralde, "A los 100 años del Electrocardiógrafo de Einthoven", Gac Med Mex, vol. 137, núm. 5, pp. 479-484, 2001.

[8] B.D. Mar, "The history of clinical Holter Monitoring”. Annals of Noninvasive Electrocardiology, vol. 10, núm. 2, pp. 226-230, 2005.

[9] L. Graindorge; A. Amblard; C. Henry; M. Limousin; L.D. Laporte; A. Hernández \& J.L Pépin, U.S. Patent Application 14/617,534, 2015.

[10] S. Horner \& P.B. Crilly, "A review of fetal ECG detection and enhancement methodologies", System Theory, Proceedings. Twenty-Third Southeastern Symposium, University of South Carolina, Columbia, South Carolina, Estados Unidos, pp. 278-282, Marzo 1991.

[11] A. Haeberlin, et al. "The optimal lead insertion depth for esophageal ECG recordings with respect to atrial signal quality". Journal of electrocardiology, vol. 46, núm. 2, pp. 158-165. 2013

[12] Yong Kyu Lim; KoKeun Kim; Kwang Suk Park, "The ECG Measurement in the Bathtub Using the Insulated Electrodes", Engineering in Medicine and Biology Society, 2004. IEMBS '04. 26th Annual International Conference of the IEEE, vol.1, pp. 2383-2385, Septiembre 2004.

[13] Xiaofei Pu; Lei Wan; Yun Sheng; Chiang, P.; Yajie Qin; Zhiliang Hong, “A Wireless 8-Channel ECG Biopotential Acquisition System for dry Electrodes", Radio-Frequency Integration Technology (RFIT), 2012 IEEE International Symposium, pp. 140-142, Noviembre 2012.

[14] J. Reynolds, “Electrocardiografía del corazón de la ballena”. Acta MedColomb, vol. 18, núm. 2, 1993.

[15] I. Escobar-Mejía, I. "Para recordar los inicios de la ingeniería biomédica en Antioquia", Revista Ingeniería Biomédica, 2014. 
[16] M. Pinto; J.G.B. Pérez; O.L. Rueda; M.P. Aparicio \& J.G. Barajas,

“Cardiotacómetro digital prototipo para estudio de la variabilidad de la frecuencia cardíaca", Revista Uis Ingenierías, vol. 2, núm. 2, 2011.

[17] W.J. Tompkins \& M.L. Ahlstrom, “Digital Filters for Real-Time ECG Signal Processing Using Microprocessors", Biomedical Engineering, IEEE Transactions, vol. BME-32, núm. 9, pp.708-713, Septiembre 1985.

[18] V.X. Afonso; W.J. Tompkins; T.Q. Nguyen \& L. Shen, “ECG beat detection using filter banks", Biomedical Engineering, IEEE Transactions, vol.46, núm. 2, pp.192-202, Febrero 1999.

[19] S.C. Bera; R. Sarkar \& N. Mandal, "A Review Work on Reconstruction of ECG Wave from Fourier Harmonic Components", Industrial and Information Systems, 2008. ICIIS 2008. IEEE Region 10 and the Third international Conference, pp. 1-5, Diciembre. 2008.

[20] V. Seena \& J. Yomas, "A review on feature extraction and denoising of ECG signal using wavelet transform" Devices, Circuits and Systems (ICDCS), 2014 2nd International Conference, pp. 1-6, Marzo 2014.

[21] J. P. Barrero; J. B. González \& M. Pinto, “Cardiotacómetro Digital Prototipo para Estudio de la V de la Frecuencia Cardíaca". Revista UIS Ingenierías, vol. 2, núm. 2, pp. 81-86, 2011.

[22] J. P. Barrero \& J. B. González, Implementación de filtros adaptativos en DSP aplicados al tratamiento de interferencia de $60 \mathrm{hz}$ y desplazamiento de la línea de base del ECG, 2011

[23] L.O. Sarmiento; A González \& J. Millet, "Synthetic database for testing algorithms of fetal ECG extraction from abdominal ECG". In Image, Signal Processing, and Artificial Vision (STSIVA), 2012 XVII Symposium, pp. 56-61. Septiembre 2012.

[24] J. González; R.M. Gutiérrez; M.O. Tamayo \& L. Gómez, “Construcción y análisis no lineal de bases de datos de electrocardiogramas de felinos". En De Señales, Imágenes y Visión Artificial, vol. 1, pp. 58$62,2008$. 
[25] A. Hurtado \& A. Salamanca, Proyectos de Investigación Universidad Santo Tomás. Catálogo Fodein. Fondo de Investigaciones. Colombia, Ediciones USTA, 2015.

[26] J.E. González-Barajas, "Threshold Calculation for R Wave Detection in Complex Cardiac". Tecno Lógicas, vol. 17, núm. 32, pp. 47-55, 2014.

[27] E. Forero; J.E. González-Barajas \& I. Marino-Martínez, “Tratamiento de rizados en la estimación de la densidad espectral de potencia de la señal de ritmo cardíaco", Revista Uis Ingenierías, vol. 12, p. 2, 2014.

[28] J.E. González-Barajas \& C. Velandia-Cárdenas \& J. Nieto-Camacho, "Implementación de filtro digital en tiempo real para detección de la onda R". Tecno Lógicas, vol. 18, núm. 34, pp. 75-86, 2015.

[29] J. Enderle \& S. Blanchard, Introduction to Biomedical Engineering. USA, Academic Press Ed., p 193, 2000.

[30] S. Mitra S, Procesamiento de señales digitales. México, D.F., Mc Graw Hill, p. 420, 2007.

[31] J. González \& J. Barrero, “Implementación de filtros adaptativos en DSP aplicados al tratamiento de interferencia de $60 \mathrm{hz}$ y desplazamiento de la línea de base del ECG", Revista Energía y Computación. Colombia, Univalle, vol. 13, núm. 2, 2005.

[32] Physionet. Data base. Http//: www.physionet.org. Consultada en Septiembre de 2015.

[33] W. Tompkins, Biomedical Digital Signal Processing. USA, Prentice Hall, pp. 368, 1993.

[34] V. Solo \& K. Xuang, Adaptive Signal Processing Algorithms, USA, Prentice Hall, pp. 13 - 177, 1995.

[35] J. González, "Método de regresión polinomial aplicado a la estimación de la señal de referencia de un sistema de filtrado adaptativo por cancelación para el tratamiento del desplazamiento de la línea de base del electrocardiograma". INGE@UAN, vol. 1, núm. 1. pp. 70-79, 2009.

[36] J. López \& A. Fernández, Fisiología del ejercicio. Colombia, Panamericana, pp. 321-340, 2008. 
[37] D. Montenegro, J. González \& E. Forero. Procesamiento Digital de Perturbaciones de Calidad de Potencia Eléctrica. Universidad Santo Tomás Ed, Bogotá, Colombia, pp. 148:23-29, 2013. 



\section{ÍNDICE ANALÍTICO}

Acondicionamiento 19, 20, 34, 35, 37, 38, 39, 54, 72, 105

Adaptativo 16, 57, 58, 59, 61, 63, 64, 69, 103, 106

Amplificador de instrumentación 35, 36, 37, 40

Banco de filtros 21, 75, 76, 77

Cancelación 16, 47, 48, 49, 50, 55, 67, 68, 69

Derivadores 82

Ecuación en diferencia 55, 59

Electrocardiografía 23, 29

Electrodos 24, 25, 27, 36, 40

Filtro analógico 38

Línea de base 16, 21, 29, 41, 44, 45, 46, 47, 48, 49, 64, 65, 66, 68, 72, 103

Manejador de pierna derecha 36, 37

Muesca 51, 53, 54, 61

Nodo auriculoventricular 34

Nodo sinusal 34

Onda P 19, 33

Onda Q 19, 33, 75 
Onda R 15, 16, 17, 19, 21, 28, 30, 33, 71, 72, 73, 74, 75, 77, 78, 82, 84, 85, 87 , $89,90,91,93,103,104$

Onda S 19, 33, 75

Pesos 59

Potencia eléctrica 21, 50, 51, 52, 59, 60, 61, 63, 68, 78, 103

Promedios 65, 66, 67

Regla de adaptación 59

Regresión 64

Ritmo 21, 22, 89, 90, 93, 94, 95, 96, 97, 98, 99, 100, 101, 102, 104

Sistema FIR 42, 43, 44, 45, 47, 53, 54, 55, 57, 58, 60, 61, 62, 63, 64

Sombrero mexicano $78,79,80,81$

Suavizado 43, 83, 84

Técnica ventana 42, 43, 65

Tendencia 48, 49, 95

Transformada Z 43, 55

Umbral 73, 85, 86, 87, 88, 89

Wavelet 29, 78, 79, 80, 81, 82 



$$
\mid
$$

Esta obra se editó en Ediciones USTA, Departamento Editorial de la Universidad Santo Tomás. Se usó papel esmaltado de 300 gramos para la carátula y papel bond beige de 70 gramos para páginas internas. Tipografía: Soberana Texto. Impreso por Digiprint Editores S.A.S. 
TIEMPO ATRÁS, MUCHOS DE LOS PARÁMETROS EXTRAídOS de un registro electrocardiográfico eran calculados y analizados visualmente sobre una impresión en papel térmico. Gracias a los avances aportados por la electrónica, ahora es posible almacenar muchos registros en un formato digital, lo que permite que se generen algoritmos especializados en facilitar y hacer más rápido el análisis de señales electrocardiográficas. Este grupo de algoritmos ha sido fortalecido gracias a una nueva área de la ingeniería denominada: tratamiento digital de señales, la cual ha tenido impacto en el sector salud gracias a sus estrategias para detectar y cuantificar eventos en el monitoreo de señales electrocardiográficas. Por eso, esta obra está orientada a ilustrar las bases para automatizar procesos de cuantificación de parámetros extraídos durante el monitoreo de un paciente a través del electrocardiograma. El principal parámetro tomado en cuenta corresponde a la frecuencia cardiaca instantánea, por lo cual se deben tener en cuenta los conceptos básicos de la adquisición de la señal electrocardiográfica y su cronología.

El tratamiento digital de señales ha pasado de ser una asignatura más de los programas de ingeniería a convertirse en un interesante sector industrial en países del primer mundo. Por esto, el presente libro está dirigido a entusiasmar a un público lector que desee explorar aplicaciones de esta área al estudio de señales electrofisiológicas, como es el caso del electrocardiograma.

En la actualidad existen muchos textos que explican la teoría del tratamiento digital de señales con numerosos ejercicios que permiten al lector tener una apropiación de este tema. Lamentablemente, estos textos solo llegan hasta el concepto y muy pocos logran generar una expectativa de aplicación. El libro Electrocardiograma desde una visión digital permite al lector obtener un sentido práctico del tratamiento digital de señales y los conceptos para generar aplicaciones orientadas al estudio de señales electrocardiográficas. 